Supporting Information for

\title{
Road salt impacts freshwater zooplankton at concentrations below current water quality guidelines
}

\author{
Shelley E. Arnott, Martha P. Celis-Salgado, Robin E. Valleau, \\ Anna M. DeSellas, Andrew M. Paterson, Norman D. Yan, John P. \\ Smol, James A. Rusak
}

Tables S1-S9

Figures S1-S4

14 pages 
Table S1: Chloride concentration ( $\mathrm{mg} \mathrm{Cl} / \mathrm{L}$ ) in test media for 21-day life-history bioassay. Nominal Chloride

\begin{tabular}{ll}
\hline 0.39 (FLAMES media) & 0.39 \\
10 & 5.47 \\
25 & 20.5 \\
50 & 40 \\
75 & 70.45 \\
100 & 95.49 \\
150 & 145.46
\end{tabular}


Table S2: Models and AICs for total reproduction, considering zero-inflated and count-based components of the model. Bold indicates best model based on lowest AIC. The total number of neonates produced in 21 days is represented by 'totneon'.

\begin{tabular}{lcc}
\hline Model & df & AIC \\
\hline totneon $\sim$ species*chloride $\mid$ species*chloride & $\mathbf{2 5}$ & $\mathbf{1 7 2 0 . 0}$ \\
totneon $\sim$ species*chloride $\mid$ species+chloride & 20 & 1735.9 \\
totneon $\sim$ species*chloride $\mid$ chloride & 15 & 1778.4 \\
totneon $\sim$ species*chloride $\mid$ species & 19 & 1754.9 \\
totneon $\sim$ species*chloride $\mid 1$ & 14 & 1782.2 \\
totneon $\sim$ species+chloride $\mid$ species*chloride & 20 & 1736.2 \\
totneon $\sim$ chloride $\mid$ species*chloride & 15 & 1858.2 \\
totneon $\sim$ species $\mid$ species*chloride & 19 & 1784.4 \\
totneon $\sim 1 \mid$ species*chloride & 14 & 1873.1
\end{tabular}


Table S3: Summary statistics from best model examining the effect of chloride and species identity on total reproduction. Full model - zeroinfl(formula $=$ totneon $\sim$ species $*$ chloride species * chloride, dist $=$ "negbin"). The zero-inflated model statistically separate reproduction into two components. The first component of reproduction that comes from the number of neonates born. The second component is the zero-inflated process associated with no reproduction. Note that $D$. catawba represents the baseline condition in the comparisons below.

Count model coefficients (negbin with log link):

\begin{tabular}{lccc}
\hline & Estimate & z value & P-value \\
\hline intercept & 2.54 & 18.54 & $<0.001$ \\
D. mendotae & $\mathbf{- 0 . 7 8 5}$ & $\mathbf{- 3 . 5 6 6}$ & $<\mathbf{0 . 0 0 1}$ \\
D. minnehaha & 0.110 & 0.558 & 0.577 \\
D. pulicaria & $\mathbf{0 . 7 0 5}$ & $\mathbf{4 . 2 1 8}$ & $<\mathbf{0 . 0 0 1}$ \\
D. pulex & $\mathbf{- 0 . 3 9 6}$ & $\mathbf{- 2 . 1 5 4}$ & $\mathbf{0 . 0 3 1}$ \\
D. pulex*pulicaria & -0.27 & -1.466 & 0.143 \\
Chloride & $\mathbf{- 0 . 0 0 9}$ & $\mathbf{- 2 . 1 6 1}$ & $\mathbf{0 . 0 3 1}$ \\
D. mendotae*chloride & $\mathbf{- 0 . 0 5 6}$ & $\mathbf{- 4 . 2 4 2}$ & $<\mathbf{0 . 0 0 1}$ \\
D. minnehaha*chloride & -0.015 & -1.499 & 0.134 \\
D. pulicaria*chloride & 0.002 & 0.547 & 0.584 \\
D. pulex*chloride & 0.002 & 0.514 & 0.607 \\
D. pulex*pulicaria ${ }^{*}$ chloride & 0.001 & 0.142 & 0.887
\end{tabular}

Zero-inflation model coefficients (binomial with logit link)

\begin{tabular}{lccc}
\hline & Estimate & $\mathrm{Z}$ value & P-value \\
\hline intercept & -0.711 & -1.744 & 0.081 \\
D. mendotae & -0.402 & -0.559 & 0.576 \\
D. minnehaha & -0.182 & -0.292 & 0.771 \\
D. pulicaria & $\mathbf{- 1 . 8 1 6}$ & $\mathbf{- 2 . 4 7 6}$ & $\mathbf{0 . 0 1 3}$ \\
D. pulex & -0.099 & -0.173 & 0.863 \\
D. pulex*pulicaria & -0.465 & -0.805 & 0.421 \\
Chloride & $\mathbf{0 . 0 3 3}$ & $\mathbf{3 . 4 7 9}$ & $\mathbf{0 . 0 0 1}$ \\
D. mendotae*chloride & -0.039 & -1.200 & 0.230 \\
D. minnehaha*chloride & 0.032 & 1.406 & 0.160 \\
D. pulicaria*chloride & -0.022 & -1.874 & 0.061 \\
D. pulex *chloride & $\mathbf{- 0 . 0 3 8}$ & $\mathbf{- 3 . 2 7 0}$ & $\mathbf{0 . 0 0 1}$ \\
D. pulex*pulicaria *chloride & -0.017 & -1.521 & 0.128
\end{tabular}


Table S4: Models and AICs for mean clutch size. Bold indicates best model based on the lowest AIC. When multiple models were within 2 AIC, the most parsimonious model was chosen as the best model.

\begin{tabular}{lcc}
\hline Model & df & AIC \\
\hline clutch $\sim$ chloride*Species & 13 & 483.410 \\
clutch $\sim$ chloride+Species & $\mathbf{8}$ & $\mathbf{4 8 2 . 4 1 9}$ \\
clutch $\sim$ chloride & 3 & 548.418 \\
clutch $\sim$ Species & 7 & 501.793
\end{tabular}


Table S5: Mean clutch size and standard deviation (SD) of each species. TukeyHSD pairwise comparisons of mean clutch size among species are indicated by letters where similar letters indicate no statistical difference $(\mathrm{P}<0.05)$.

\begin{tabular}{lccc}
\hline Species & TukeyHSD & Mean clutch size & SD \\
\hline D. pulicaria & $\mathrm{a}$ & 5.18 & 1.26 \\
D. catawba & $\mathrm{ab}$ & 4.11 & 1.75 \\
D. pulicaria ${ }^{\text {pulex hybrid }}$ & $\mathrm{b}$ & 3.88 & 2.68 \\
D. minnehaha & $\mathrm{b}$ & 3.74 & 1.96 \\
D. pulex & $\mathrm{b}$ & 3.42 & 1.63 \\
D. mendotae & $\mathrm{c}$ & 2.00 & 1.23
\end{tabular}


Table S6: Models and AICs for mean day of first reproduction. Bold indicates best model based on AIC. When multiple models were within 2 AIC, the most parsimonious model was chosen as the best model.

\begin{tabular}{lcc}
\hline Model & df & AIC \\
\hline DOFR $\sim$ chloride*Species & 13 & 944.13 \\
DOFR $\sim$ chloride+Species & 8 & 943.95 \\
DOFR $\sim$ chloride & 3 & 1084.16 \\
DOFR $\sim$ Species & 7 & $\mathbf{9 4 2 . 7 7}$
\end{tabular}


Table S7: Models and AICs for daily mortality rate. Bold indicates best model based on AIC.

\begin{tabular}{lcc}
\hline Model & df & AIC \\
\hline mortality $\sim$ chloride $*$ Species & $\mathbf{1 3}$ & $\mathbf{- 1 8 2 . 5 8}$ \\
mortality $\sim$ chloride + Species & 8 & -153.30 \\
mortality $\sim$ chloride & 3 & -142.60 \\
mortality $\sim$ Species & 7 & -115.83
\end{tabular}


Table S8: Comparison of chloride-mortality relationship for each species. "Slope com" represents results of pairwise comparisons of slopes where species with statistically significantly different slopes (based on FDR adjusted p-values that are $<0.05$ ) are indicated by different letters. P-values adjusted for false discovery rate (FDR adjusted) indicate slopes significantly different from 0 . Statistically significant values are bold.

\begin{tabular}{lccccc}
\hline Species & Slope com & slope & $95 \%$ LCI & $95 \%$ UCI & $\begin{array}{c}\text { P (FDR } \\
\text { adjusted) }\end{array}$ \\
\hline D. pulex & a & 0.0002 & -0.00018 & 0.00057 & 0.297 \\
Hybrid & a & $\mathbf{0 . 0 0 0 4 2}$ & $\mathbf{0 . 0 0 0 0 5}$ & $\mathbf{0 . 0 0 0 8 0}$ & $\mathbf{0 . 0 3 5}$ \\
D. pulicaria & $\mathbf{a}$ & $\mathbf{0 . 0 0 0 4 3}$ & $\mathbf{0 . 0 0 0 0 6}$ & $\mathbf{0 . 0 0 0 8 1}$ & $\mathbf{0 . 0 3 5}$ \\
D. catawba & b & $\mathbf{0 . 0 0 1 0 5}$ & $\mathbf{0 . 0 0 0 6 8}$ & $\mathbf{0 . 0 0 1 4 3}$ & $<\mathbf{0 . 0 0 1}$ \\
D. minnehaha & bc & $\mathbf{0 . 0 0 1 1 6}$ & $\mathbf{0 . 0 0 0 7 9}$ & $\mathbf{0 . 0 0 1 5 4}$ & $<\mathbf{0 . 0 0 1}$ \\
D. mendotae & $\mathbf{c}$ & $\mathbf{0 . 0 0 1 6 6}$ & $\mathbf{0 . 0 0 1 2 8}$ & $\mathbf{0 . 0 0 2 0 3}$ & $<\mathbf{0 . 0 0 1}$
\end{tabular}


Table S9. Effect of chloride on $r$, intrinsic rate of population growth. Comparison of slopes ( $r$ versus chloride) for species, including lower and upper $95 \%$ confidence intervals and $p$-values adjusted for a false discovery rate of $5 \%$. Bold indicates a significant effect of chloride on $r$. "Slope com" represents the results of paired comparisons between slopes with similar letters indicating no difference in slopes (adjusted to control false discovery rate; FDR-adjusted).

\begin{tabular}{lccccc}
\hline Species & Slope com & slope & $95 \%$ LCI & $95 \%$ UCI & P (FDR-adjusted) \\
\hline D. minnehaha & a & $\mathbf{- 0 . 0 0 3}$ & $\mathbf{- 0 . 0 0 4}$ & $\mathbf{- 0 . 0 0 3}$ & $<\mathbf{0 . 0 0 1}$ \\
D. mendotae & ab & $\mathbf{- 0 . 0 0 3}$ & $\mathbf{- 0 . 0 0 4}$ & $\mathbf{- 0 . 0 0 4}$ & $\mathbf{0 . 0 0 1}$ \\
D. catawba & bc & $\mathbf{- 0 . 0 0 1}$ & $\mathbf{- 0 . 0 0 2}$ & $\mathbf{- 0 . 0 0 0 2}$ & $\mathbf{0 . 0 2 5}$ \\
Hybrid & c & $\mathbf{- 0 . 0 0 0 9}$ & $\mathbf{- 0 . 0 0 1}$ & $\mathbf{- 0 . 0 0 0 3}$ & $\mathbf{0 . 0 0 6}$ \\
D. pulicaria & c & -0.0005 & -0.0003 & 0.00006 & 0.091 \\
D. pulex & c & -0.0003 & -0.0008 & 0.0003 & 0.313
\end{tabular}


Figures

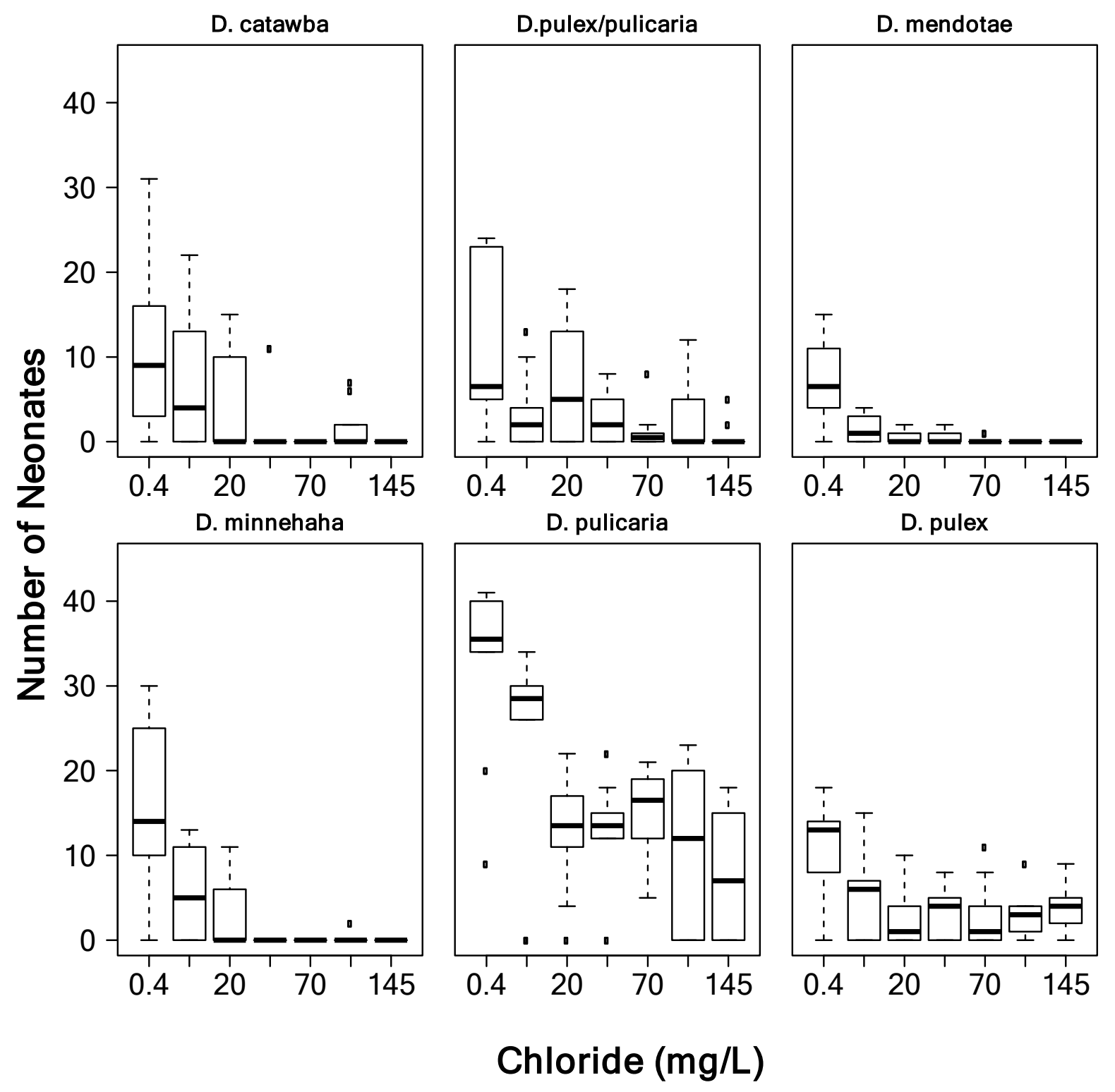

Figure S1: Total number of neonates produced by each individual Daphnia during the 21-day bioassay. Chloride concentrations represent the actual, measured chloride concentration the test media. $\mathrm{N}=10$ individuals for each species and chloride treatment. The center line represents the median and the box represents the $1^{\text {st }}$ and $3^{\text {rd }}$ quartile. Upper whiskers represent the interquartile range times the smaller of the maximum value for individual mean clutch size and the $3^{\text {rd }}$ quartile. Lower whiskers represent the interquartile range times the larger of the minimum value for individual mean clutch size and the $1^{\text {st }}$ quartile. 

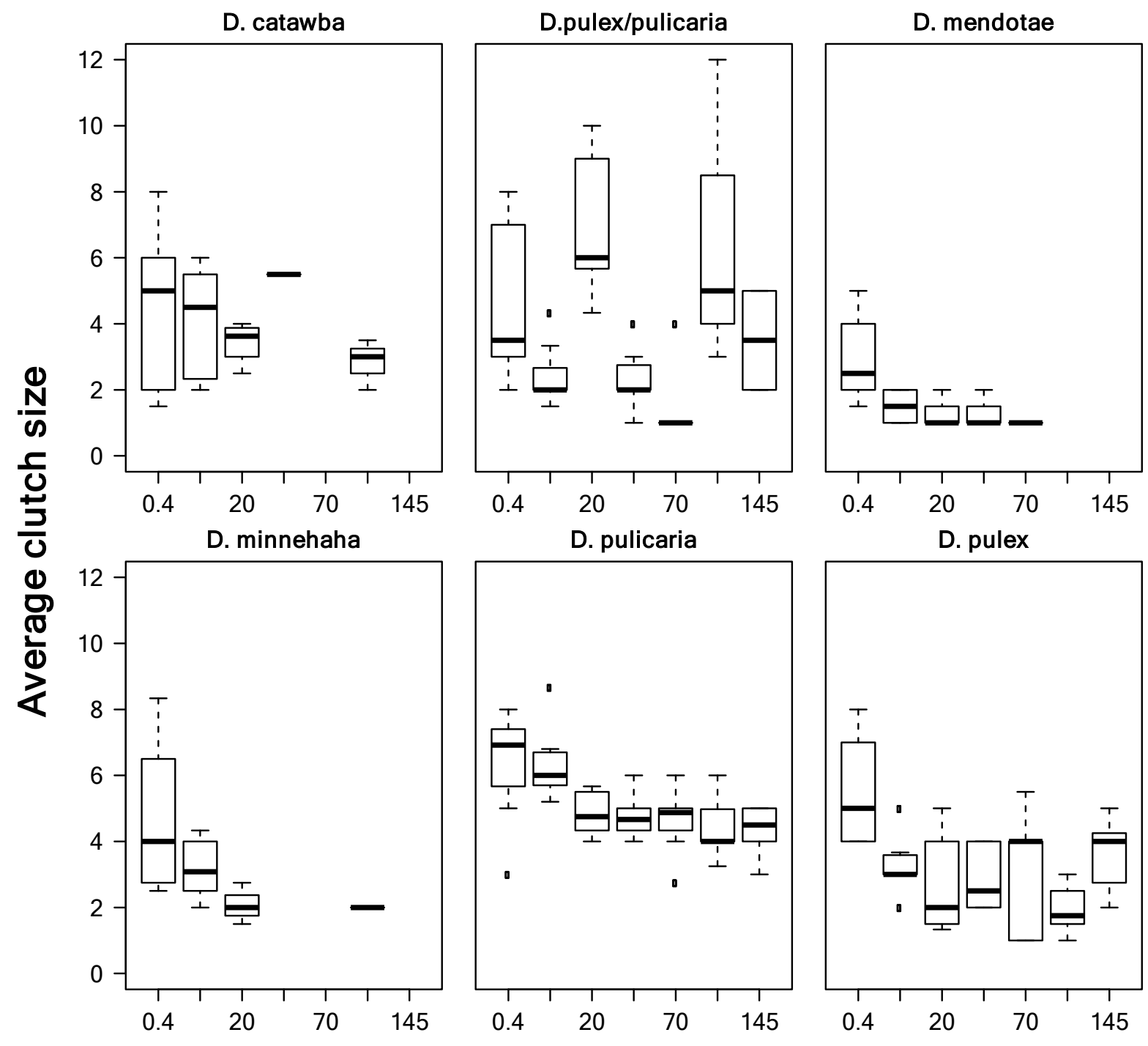

Chloride (mg/L)

Figure S2: The average clutch size for each Daphnia individual in each treatment during the 21day experiment. The center line represents the median and the box represents the $1^{\text {st }}$ and $3^{\text {rd }}$ quartile. Upper whiskers represent the interquartile range times the smaller of the maximum value for individual mean clutch size and the $3^{\text {rd }}$ quartile. Lower whiskers represent the interquartile range times the larger of the minimum value for individual mean clutch size and the $1^{\text {st }}$ quartile. 


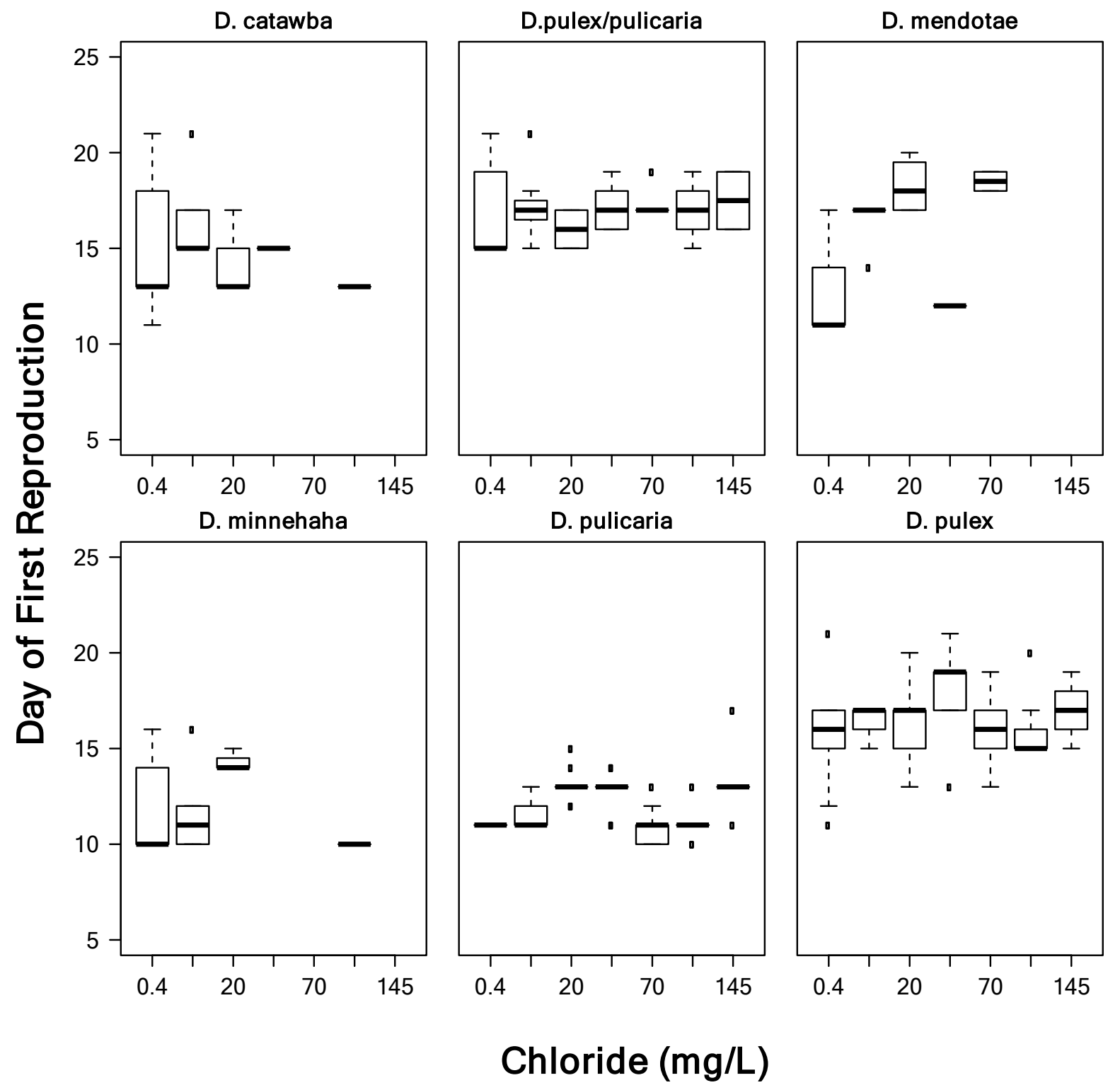

Figure S3: The day of experiment that each Daphnia reproduced for the first time. Missing values indicate where no reproduction occurred. The center line represents the median and the box represents the $1^{\text {st }}$ and $3^{\text {rd }}$ quartile. Upper whiskers represent the interquartile range times the smaller of the maximum value for individual mean clutch size and the $3^{\text {rd }}$ quartile. Lower whiskers represent the interquartile range times the larger of the minimum value for individual mean clutch size and the $1^{\text {st }}$ quartile. 


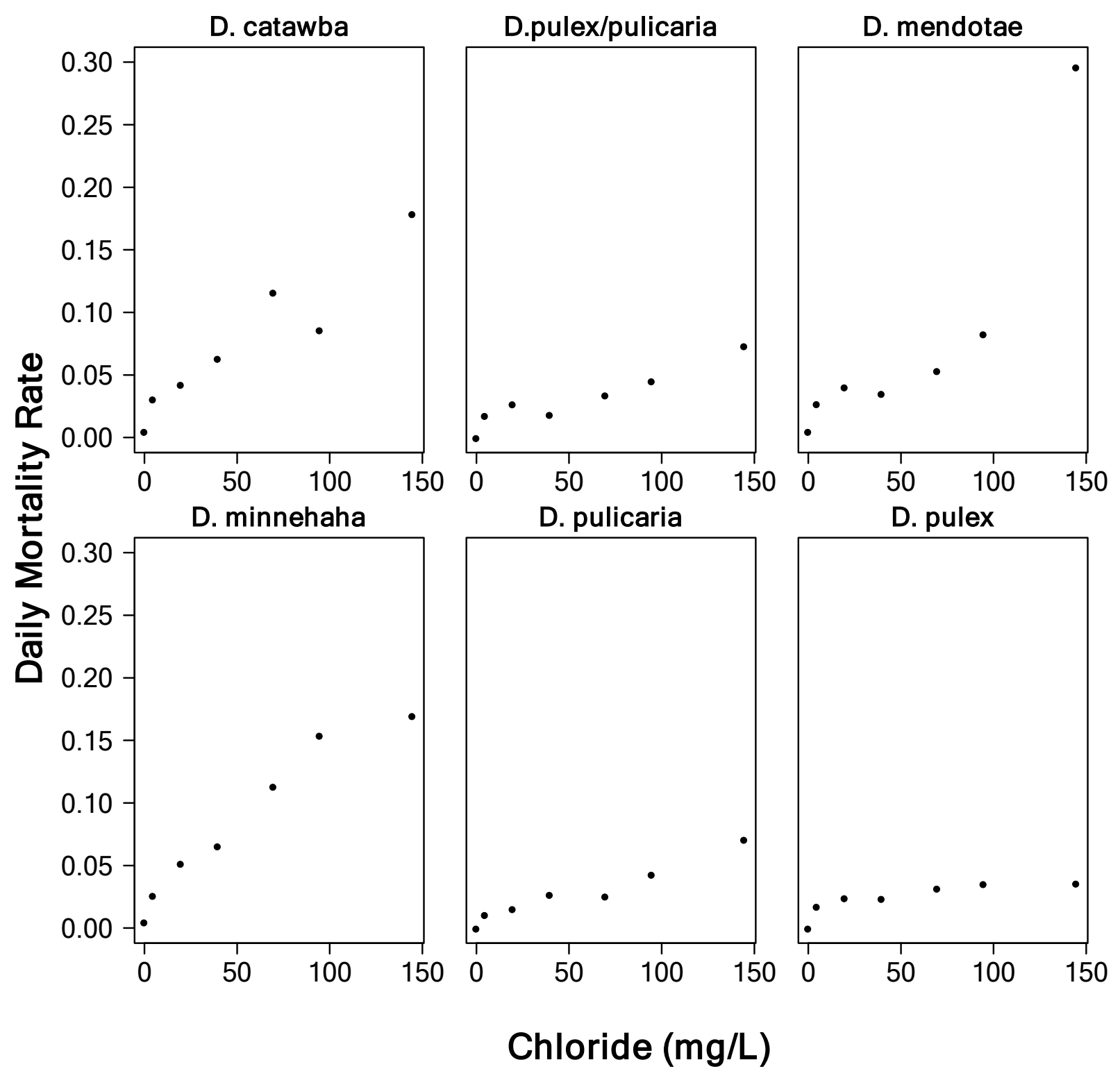

Figure S4: Daily mortality rate for each species in each of 7 chloride treatments. Daily mortality rate was estimated for each chloride concentration using a parametric regression model for interval-censored data and fitting an exponential decay with days. 